\title{
Peripartum Cardiomyopathy During Puerperium in a Pregnancy Complicated by Severe Pre-Eclampsia, HELLP Syndrome and Acute Renal Failure: A Case Report
}

\author{
Junjie Wanga, c, Lay Kok Tan ${ }^{\mathrm{b}}$
}

\begin{abstract}
We report a case of antenatal severe pre-eclampsia which was complicated by postpartum HELLP syndrome, acute kidney injury and postpartum cardiomyopathy. A 35-year-old patient in her 34th week of gestation underwent a cesarean section in view of severe pre-eclampsia. She developed HELLP syndrome postpartum, which was complicated by acute kidney injury. After the resolution of HELLP syndrome and improvement of her kidney function, she developed postpartum cardiomyopathy.
\end{abstract}

Keywords: Cardiomyopathy; Pre-eclampsia; Pregnancy

\section{Introduction}

Peripartum cardiomyopathy (PPCM) is a type of dilated cardiomyopathy of unknown origin. It occurs in previously healthy women in the final month of pregnancy and up to 5 months after delivery. Although the incidence is low, morbidity and mortality rates are high, ranging from $5 \%$ to $32 \%$. The diagnosis of this rare disease is difficult to make. Pregnancy-related pathologies such as pre-eclampsia, myocarditis, or underlying genetic disease show overlapping symptoms with PPCM. Preeclampsia is a multisystemic disorder that presents with hypertension and proteinuria. Edema is frequently present in patients with pre-eclampsia. Hypertension, proteinuria and edema are also symptoms of PPCM. Patients with severe pre-eclampsia complicated by PPCM should be managed by a multidisciplinary team of specialists to improve morbidity and mortality.

\footnotetext{
Manuscript accepted for publication October 06, 2015

aDepartment of Obstetrics and Gynaecology, KK Women's and Children's Hospital, Singapore

bDepartment of Obstetrics and Gynaecology, Singapore General Hospital, Singapore

${ }^{\mathrm{c} C o r r e s p o n d i n g ~ A u t h o r: ~ J u n j i e ~ W a n g, ~ D e p a r t m e n t ~ o f ~ O b s t e t r i c s ~ a n d ~ G y n a e c o l-~}$ ogy, KK Women's and Children's Hospital, 100 Bukit Timah Road, Singapore 229899, Singapore. Email: wgjunjie@gmail.com
}

doi: http://dx.doi.org/10.14740/jmc2025w

\section{Case Report}

Our patient is a 35-year-old woman, gravida 2, para 2, bearing a singleton pregnancy. Her first pregnancy was normal with a term delivery. She presented with first trimester hypertension and was given methyldopa but defaulted on her medication.

She was seen in outpatient clinic at 33 weeks of gestation and was noted to have a raised blood pressure but no proteinuria. Ultrasound done showed mild intrauterine growth restriction. She was admitted for observation and started on methyldopa. At 34 weeks of gestation, she presented with sudden onset epigastric pain and her blood pressure was $220 / 110 \mathrm{~mm}$ $\mathrm{Hg}$. Liver function test showed transaminitis. Oral nifedipine, intravenous hydralazine and intravenous magnesium sulphate was started and she underwent an emergency cesarean section at 34 weeks of gestation.

On postoperative day 1 , the patient developed jaundice. Blood tests showed raised bilirubin, transaminitis and low platelets. The patient also had gross hematuria. A diagnosis of HELLP syndrome was made and the patient was transfused with platelets and fresh frozen plasma. Her hemoglobin level was noted to be $7.1 \mathrm{~g} / \mathrm{dL}$. Therefore, one pint of packed cells was transfused. Her creatinine level was noted to be 341 $\mu \mathrm{mol} / \mathrm{L}$. She was seen by the renal physician and was diagnosed to have acute kidney injury secondary to HELLP syndrome.

On postoperative day 3 , she developed worsening dyspnea. Chest radiography showed left lower lobe pneumonia and pulmonary congestion. In view of rising trend of total white blood cells, a diagnosis of nosocomial pneumonia was made. She was started on intravenous broad spectrum antibiotics.

On postoperative day 5 , she had persistent and worsening dyspnea and was noted to be clinically overloaded. Chest $\mathrm{X}$-ray showed that the lungs were congested and there is bilateral perihilar airspace opacification in a bat wing's configuration, which was worrisome for pulmonary edema. She was given frusemide and a cardiologist referral was made. A two-dimensional echocardiography was done which showed left ventricular ejection fraction of $58 \%$ with moderate mitral regurgitation, unlikely to be PPCM.

Ultrasound abdomen and kidneys showed normal liver and kidney functions. Her creatinine levels and liver function tests progressively returned to normal. Fluid overload resolved with strict fluid restriction. Her pneumonia resolved after 10 
days of intravenous antibiotics. The patient was discharged on POD15 well and afebrile.

The patient returned 3 days later to the emergency department complaining of left thigh blisters with fever. She also complained of mild dyspnea and a productive cough. Her vitals showed tachycardia with a pulse rate of 124 beats per minute but blood pressure was normal. On examination, there was a $2 \mathrm{~cm}$ vesicular lesion on the anterior aspect of her left thigh. Chest X-ray showed increased opacity and haziness over bilateral lower lung lobes compared to previous X-rays. Patient was advised for admission to treat for pneumonia but she declined and went home.

She returned to the emergency department a day later with increased shortness of breath and persistent left thigh blister that was increasing in size. She was tachypneic and unable to speak in full sentences. Her blood pressure was 169/126 $\mathrm{mm} \mathrm{Hg}$ and heart rate was 158 beats per minute. The temperature was $37.5^{\circ} \mathrm{C}$. Her oxygen saturation was $35 \%$ on a non-rebreather mask. Arterial blood gas showed metabolic and respiratory acidosis with type 1 respiratory failure. Patient had a seizure while her blood was taken which was aborted with intravenous midazolam. She was subsequently intubated and kept on mechanical ventilation. Lung auscultation revealed bilateral basal crepitation. Chest X-ray showed bilateral pulmonary congestion and a diagnosis of acute pulmonary edema was made. She was then admitted to the surgical intensive care unit with central line monitoring.

The patient was reviewed by the cardiologists and a formal two-dimensional echocardiography was done. It showed left ventricular ejection fraction of $25-30 \%$, mild global left ventricular hypokinesia, moderate to severe mitral regurgitation, moderately dilated left atrium, mildly dilated right atrium and normal ventricles. A diagnosis of PPCM was made. Patient was treated with intravenous frusemide and started on broad spectrum antibiotics. Residual HELLP syndrome was unlikely as the patient was 3 weeks postpartum and biochemical investigations do not support ongoing or worsening HELLP syndrome. The patient had an episode of pulseless electrical activity 1 day after admission to intensive care unit. She was resuscitated for $3 \mathrm{~min}$ and intravenous adrenaline was given with return of spontaneous circulation within $2 \mathrm{~min}$. The patient's care was taken over by the cardiologists and she was transferred to the cardiology intensive care unit.

She was extubated 3 days after admission and intravenous frusemide was converted to oral. Anti-hypertensives were started for blood pressure control and diuresis was achieved with negative balance of up to $500 \mathrm{~mL} /$ day. Her renal function gradually improved with creatinine levels on a downward trend till normal before discharge. Infection markers were also on a downward trend and intravenous antibiotics were stopped. The patient was discharged home well 10 days after the second admission.

\section{Discussion}

Our patient first presented with HELLP syndrome secondary to severe pre-eclampsia. She was unfortunate to have suf- fered from nosocomial pneumonia due to prolonged hospital stay. Acute kidney injury was thought to be a complication of HELLP syndrome but the renal physicians did not rule out the possibility of chronic kidney disease that was not diagnosed before pregnancy. PPCM is likely, as the patient had no past history of cardiac disease before pregnancy. The patient gave a history of increased fluid intake when she was at home as she thought it would improve her kidney function. Her actions probably resulted in acute pulmonary edema as her heart was unable to cope with the increased fluid due to cardiomyopathy.

PPCM is a type of dilated cardiomyopathy of unknown origin. It occurs in previously healthy women in the final month of pregnancy and up to 5 months after delivery [1]. PPCM is rare in some places but more common in others. It seems to be more prevalent in developing countries. For example, the incidence in Nigeria $(1 \%)$ or Haiti $(0.33 \%)$ surpasses that in more developed countries, such as South Africa $(0.1 \%)$ or the United States (1:3,000 - 4,000 deliveries) [2]. The reason for these discrepancies remain unknown but may be due to environmental and genetics factor, quality of perinatal care and also socio-cultural practices.

Although the incidence is low, morbidity and mortality rates are high, ranging from $5 \%$ to $32 \%$ [3], and the diagnosis of this rare disease is not always straightforward.

PPCM is a diagnosis of exclusion and defined based on four criteria: 1) development of cardiac failure in the last month of pregnancy or within 5 months postpartum; 2) the absence of an identifiable cause for the cardiac failure; 3) absence of recognizable heart disease before the last month of pregnancy; and 4) LV systolic dysfunction shown by echocardiographic data such as depressed shortening fraction (e.g. ejection fraction less than $45 \%$, M-mode fractional shortening less than $30 \%$, or both, and an LV end-diastolic dimension of more than $2.7 \mathrm{~cm} / \mathrm{m}^{2}$ ) [4].

It may be difficult to diagnose because the symptoms are similar to those of normal late pregnancy. Giddiness, shortness of breath, fatigue, or pedal edema can happen in both normal late pregnancy and early congestive heart failure. Therefore, we need to be extremely cautious when dealing with patients with dyspnea in late pregnancy or postpartum. Careful history taking and physical examination need to be undertaken to exclude conditions that can cause or exacerbate heart failure. Volume status of the patient needs to be assessed through postural blood pressure, weight, blood investigations and physical examination. A complete works of blood investigations should be done to exclude causes of heart failure. Twelve-lead electrocardiogram and chest radiograph should be done to assess for cardiac abnormalities and hypertrophy of the heart. Chest radiographs can also be used to look for pulmonary edema. Two-dimensional echocardiogram is useful to assess LV size, wall thickness, and valvular function.

Several etiologies have been postulated but none is definite. They include viral myocarditis, abnormal immune response to pregnancy, stress activated cytokines, abnormal responses to hemodynamic stress of pregnancy, increased myocyte apoptosis, malnutrition, genetics factors, abnormal prolactin production, abnormal hormonal function, increased adrenergic tone secondary to emotional or physical stress, and myocardial ischemia. 
Risk factors include advanced maternal age, multiparity, obesity, African origin, multiple pregnancy, malnutrition, gestational hypertension, diabetes, prolonged tocolysis, low socioeconomic status, poor perinatal care, breast feeding, substance and tobacco abuse, and family history $[2,5]$.

The standard principles of acute treatment for PPCM are the same as for heart failure. This includes fluid and salt restriction, treatment of hypertension, diuretics, vasodilators and digoxin. The aim of the treatment is to relieve the symptoms, delay progression of left ventricular dysfunction, and improving survival. Special cautions need to be given when deciding which medications when given to the mother will be harmful to the neonate when breastfeeding. Currently, cardiologists use three types of drugs (diuretics, angiotensin-converting enzyme inhibitors or angiotensin receptor blockers, and B-blockers) for the treatment of chronic heart failure [6].

Diuretics help to improve pulmonary and peripheral edema within hours or days [6]. ACEIs improve survival in all severities of myocardial disease, but are contraindicated in pregnancy and breastfeeding, due to its severe teratogenicity. ARBs help to improve survival in patients with heart failure but they have similar teratogenic risks as ACEIs. Three different B-blockers (metoprolol, carvedilol and bisoprolol) have been shown to reduce mortality in heart failure [6].

Atrial arrhythmias should be treated with digoxin. However, class III and class IV anti-arrhythmias should be avoided because of their teratogenic risks [7].

Early delivery is recommended if the condition of a patient with PPCM is unstable despite medical treatment. Prostaglandins and oxytocin can be used as methods of inducing labor with minimal risks. Adequate analgesia, such as epidural, is recommended to reduce the load of the heart as pain will stimulate a sympathetic response. Strict fluid restriction should be maintained in order to avoid fluid overload. It will be beneficial to insert an indwelling urine catheter or even a central line to monitor the volume status of the patient. A vaginal delivery is recommended over cesarean section in view of the potential surgical risks of cesarean section. However, a cesarean delivery should be performed if there are obstetrics indications. Thromboprophylaxis should be given peripartum.

Pre-eclampsia is a multisystem condition, involving hypertension and proteinuria, which happens in pregnancy after 20 weeks of gestation. Edema is frequently present. The pathophysiology is multifactorial and involves endothelial damage and capillary leak. Some of the signs and symptoms of pre-eclampsia include headache or visual disturbances, impaired coagulation, impaired liver function, pulmonary edema, ascites, and renal failure. Pulmonary edema, ascites and proteinuria are caused by endothelial dysfunction with subsequent capillary leak. As pulmonary edema, ascites and proteinuria occur in heart failure too, it makes the diagnosis of PPCM difficult [8].

HELLP syndrome is a severe complication of pre-eclampsia and it involves hemolysis, elevated liver enzymes and thrombocytopenia [9]. It occurs in $<1 \%$ of pregnancies dur- ing the third trimester or in the first few days postpartum. The pathophysiology involves microangiopathic hemolytic anemia secondary to general activation of coagulation cascade. It presents with epigastric pain, visual disturbances, headache and parasthesiae. Disseminated intravascular coagulation is the most frequent major complication. Acute renal failure occurs in almost half of such cases.

Our patient's encounter demonstrates the complexity of managing a patient with severe pre-eclampsia complicated by HELLP syndrome and acute renal failure, and PPCM. The symptoms are often similar which makes the diagnosis extremely difficult. A multidisciplinary team of specialists will be required for optimal care and management of such patients.

\section{Grant}

None.

\section{Conflict of Interest}

None.

\section{References}

1. Hibbard JU, Lindheimer M, Lang RM. A modified definition for peripartum cardiomyopathy and prognosis based on echocardiography. Obstet Gynecol. 1999;94(2):311316.

2. Karaye KM, Henein MY. Peripartum cardiomyopathy: a review article. Int J Cardiol. 2013;164(1):33-38.

3. Tidswell M. Peripartum cardiomyopathy. Crit Care Clin. 2004;20(4):777-788, xi.

4. Demakis JG, Rahimtoola SH, Sutton GC, Meadows WR, Szanto PB, Tobin JR, Gunnar RM. Natural course of peripartum cardiomyopathy. Circulation. 1971;44(6):10531061.

5. Cunningham C, Rivera J, Spence D. Severe preeclampsia, pulmonary edema, and peripartum cardiomyopathy in a primigravida patient. AANA J. 2011;79(3):249-255.

6. Cruz MO, Briller J, Hibbard JU. Update on peripartum cardiomyopathy. Obstet Gynecol Clin North Am. 2010;37(2):283-303.

7. Page RL. Treatment of arrhythmias during pregnancy. Am Heart J. 1995;130(4):871-876.

8. Sibai BM, Stella CL. Diagnosis and management of atypical preeclampsia-eclampsia. Am J Obstet Gynecol. 2009;200(5):481 e481-487.

9. Redzko S, Przepiesc J, Urban J. Hemolysis, elevated liver enzymes, and low platelets syndrome, peripartal cardiomyopathy and disseminated intravascular coagulation during the puerperium. Eur J Obstet Gynecol Reprod Biol. 2005;121(1):120-123. 